\title{
தமிழும் சோதிடமும்
}

\section{Tamil and Astrology}

\author{
திலக். கி. பாஸ்கரன் / Thilak.K.Baskaran ${ }^{1}$ \\ பொறியாளர் சு. சிவராம்கிருஷ்ணன் / Er.S.Sivaramkrishnan ${ }^{2}$
}

\begin{abstract}
Tamil language is a classical language and is one of the longest surviving classical languages. Recent archeology findings reveal that Tamil civilization was the oldest one. The trace of Tamil is seen all over the world. Also the root word analysis indicates that the most of the languages could not find its meaning in their language but makes correct meaning in Tamil language. Therefore it is clear that Tamil

Date of submission: 2019-05-01 Date of acceptance: 2019-07-02 Date of Publication: 2019-07-30 Corresponding author's Name: Thilak K. Baskaran Email: baskaraastrology@yahoo.in is the mother of all the languages. Tamil has been developed with 3 sangam (Era). The time of third (last) sangam period is said to be BC500 and could even be earlier. Therefore, the first and second sangam period could have been even older. Tholkappiyam is a work on the grammar of the Tamil language and the earliest extant work of Tamil literature and linguistics. The period at which the Tamil language had Grammar proves the antiquity and beauty of Tamil language. Tamil language evolved naturally. Tamil people's life is completely based on the nature (Planets-Astrology). The knowledge and understanding of the ancient Tamil people about Nature - Planetary position (Astrology) is astonishing. Their festivals, rituals, life style, customs etc are evident that their life was completely based on the nature. The language that evolved naturally and the civilization that grew on the basis of nature should have similarity in it. This article explores such similarity and brings out how Tamil and their civilization are similar in their concepts. It also proves that the concepts evolved based on nature will have unique similarity.
\end{abstract}

Key Words: Tamil and Astrology, Tamils life style, Astrology, Nature, Tholkappiyam, Planets, Lemuria, Kumari kandam.

\section{முன்னுரை}

தமிழ் என்ற மொழி, இயற்கையாக உருவான மொழி (Victor, 2012, p.97). தமிழர்களின் வாழ்வியலும், இயற்கையை தழுவிய வாழ்வியலே (Victor, 2012, p.141) என்ற அடிப்படையில் உருவான கருதுகோள் தான், “தமிழும் சோதிடமும்“ என்ற இந்த அறிய ஒப்புமை படைப்பு. தமிழையும் தமி ழர் களை யும் பெருமைக்கொள் ள
வைக்கும் இன்னும் ஓர் சான்று.

தமிழ் என்ற மொழி இயற்கையான மொழி (Devaneya Pavanar, 2018, p.170). சோதிடம் என்பது இயற்கையை அறிய உதவும் கருவி. இயற்கையை மையப்படுத்தி அமையும் தமிழும், சோதிடமும் ஒரே மாதிரியான கட்டமைப்பை கொண்டிருக்கும் என்பதற்கு இந்த கட்டுரை சாலச்சிறந்த சான்று.

\footnotetext{
${ }^{1}$ The author is a inventor of Baskara Astrology (KB system), Madurai, India, baskaraastrology@ yahoo.in

${ }^{2}$ The author is a follower of Baskara Astrology (KB system), Senior Engineer, Bangalore, India, scientificastrologics@gmail.com
} 


\section{8 / தமிழ்ப் பேராய்வு ஆய்விதழ்}

\section{காலச்சக்கரம் - ராசிமானம்}

சோதிடத்தின் அடிப்படை காலச்சக்கரம். காலச்சக்கரம் என்பது ராசமமானம். ராச மானம் எல்லாவற்றையும் தன்னகத்தே கொண்டுள்ளது. சோதிடத்தில் கூறப்படும் எந்த ஒரு விடயமும் காலச்சக்கரத்திலிருந்தே சொல்லப்படுகிறது (Baskaran, 2005, p.1).

இந்த ராசிமானத்தின் அடிப்படை 12 ராசிகள். பன்னிரண்டு ராசிகளை மேலும் பிரிக்கும் பொருட்டு அதனை சந்திரனின் சுழ்்சயை கொண்டு 27 நட்சத்திரங்களாக பிரித்தனர். இவ்வாறு பிரிக்கும் பொது அவை 18 முழு நட்சத்திரமா கவும் 9 பகிர் ந்தளிக்கப்பட்ட நட்சத்திரமாகவும் அமையும். இதனை மேலும் சர் செய்ய, நட்சத்திரத்திற்கு 4 பாதங்கள் வீதம் 108 பாதங்கள் உருவாக்கப்பட்டன. இதைப்பற்றி மேலும் பின்னர் விளக்கப்படும் (Baskaran, 2013, p.25) .

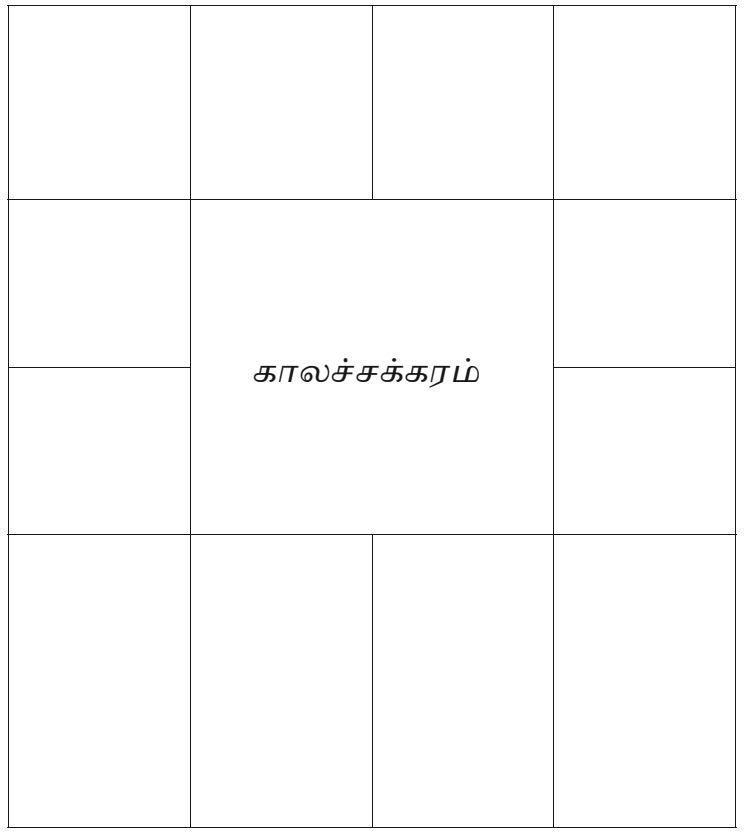

\section{ஆண் - பெண் ராசிகள்}

சோதிடத்தில் பனிரெண்டு ராசிகளையும் ஆண் மற்றும் பெண் ராச என்று இரு ராசிகளாக உருவாகியுள்ளனர் (Baskaran, 2017, p.50).

\begin{tabular}{|c|c|c|c|}
\hline பெண் & அண் & பெண் & ஆண் \\
\hline ஆண் & \multirow{2}{*}{\multicolumn{2}{|c|}{ காலச்சக்கரம் }} & பெண் \\
\hline பெண் & & & ஆண் \\
\hline ஆண் & பெண் & ஆண் & பெண் \\
\hline
\end{tabular}

\section{இயக்கங்களை குறித்த ராசிகள்}

சோதிடத்தில் சரம், ஸ்திரம், உபயம் என்ற இயக்கங்களை குறித்த ராசிகள் உள்ளன (Baskaran, 2017, p.55).

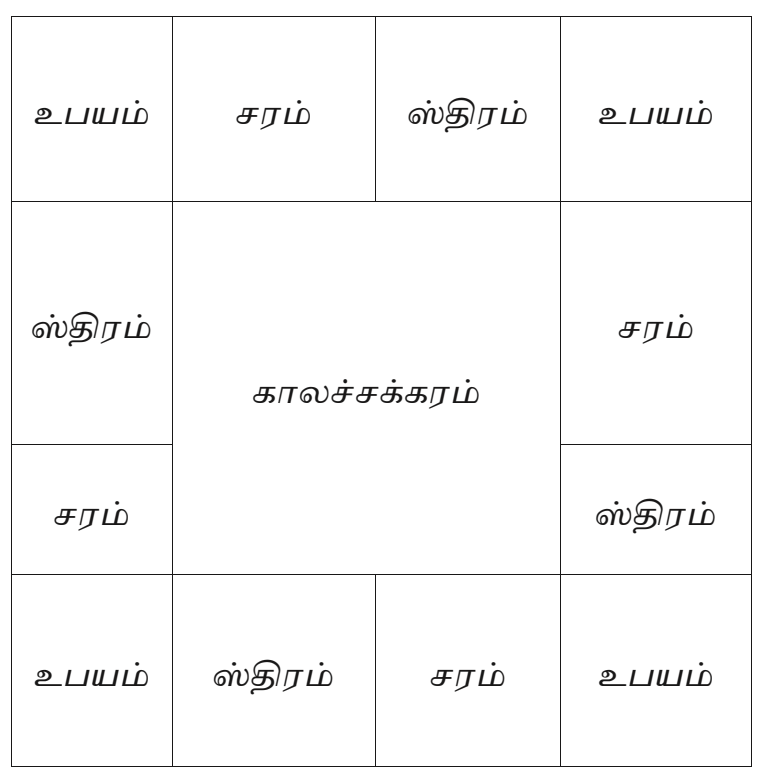

\section{உயிர்பற்றுபொருளப்பற்று என்ற ராசிகள்}

சோதிடத்தில் பன்னிரெண்டு ராசிகளையும் உயிர்பற்று (லக்னம் சார்ந்தது) மற்றும் புறம் பொருளப்பற்று (லக்னம் சாராதது) ராசி என்று இருராசுகளாக உருவாகியுள்ளனர (Baskaran, 2010, p.48). 


\section{ஐந்திணை}

சோதிடத்தின் பஞ்ச பூத தத்துவமான நெருப்பு நிலம் காற்று நீர் ஆகாயம்

\begin{tabular}{|c|c|c|c|}
\hline $\begin{array}{c}\text { பொருளப்பற்று } \\
\text { புறம் }\end{array}$ & $\begin{array}{l}\text { உயிர்பற்று } \\
\text { அகம் }\end{array}$ & $\begin{array}{c}\text { பொருளப்பற்று } \\
\text { புறம் }\end{array}$ & $\begin{array}{c}\text { பொருளப்பற்று } \\
\text { புறம் }\end{array}$ \\
\hline $\begin{array}{l}\text { உயிர்பற்று } \\
\text { அகம் }\end{array}$ & \multirow{2}{*}{\multicolumn{2}{|c|}{ காலச்சக்கரம் }} & $\begin{array}{c}\text { பொருளப்பற்று } \\
\text { புறம் }\end{array}$ \\
\hline $\begin{array}{c}\text { பொருளப்பற்று } \\
\text { புறம் }\end{array}$ & & & உயிர்பற்று அகம் \\
\hline $\begin{array}{l}\text { உயிர்பற்று } \\
\text { அகம் }\end{array}$ & $\begin{array}{c}\text { பொருளப்பற்று } \\
\text { புறம் }\end{array}$ & $\begin{array}{l}\text { உயிர்பற்று } \\
\text { அகம் }\end{array}$ & $\begin{array}{l}\text { பொருளப்பற்று } \\
\text { புறம் }\end{array}$ \\
\hline
\end{tabular}

(நட்சத்திரம்) என்பதே ராசி மனம் உருவாக இருந்த அடிப்படை காரணமாகும் (Baskaran, 2017, p.60).

\section{லக்னம்}

சோதிடம் என்பது வெளிச்சம் (சூரியன்) விழும் இடம். இதையே சோதிடத்தில் லக்னம் என்கிறோம். சோதிடத்தில் ஒருவரின் விதியை தீர்மானிப்பது லக்கினமே (Baskaran, 2013, p.23).

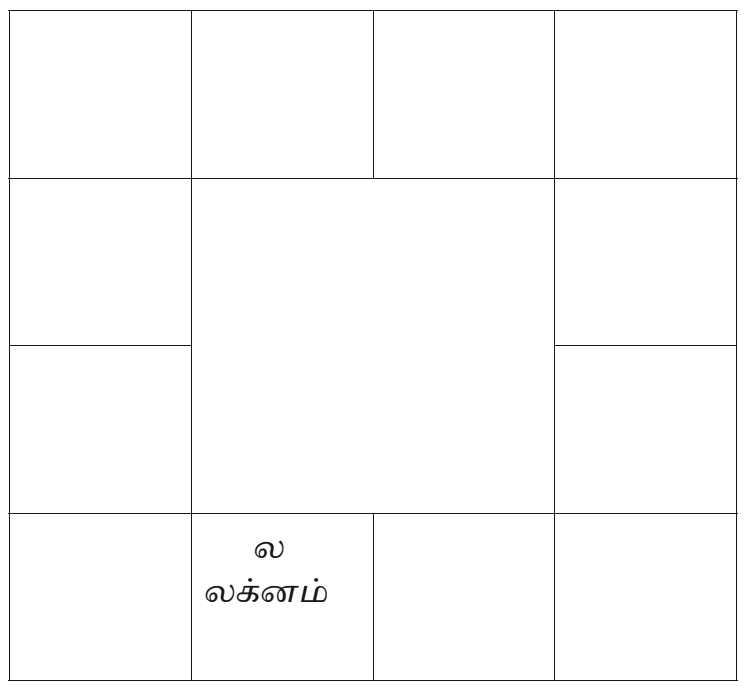

மூலம்:<http://www.baskaraastrology.co.in/>, $\mathrm{http} / / /$ www.scientificastrologyexpert.com/tamil.. html

\section{பருவ காரகங்கள்}

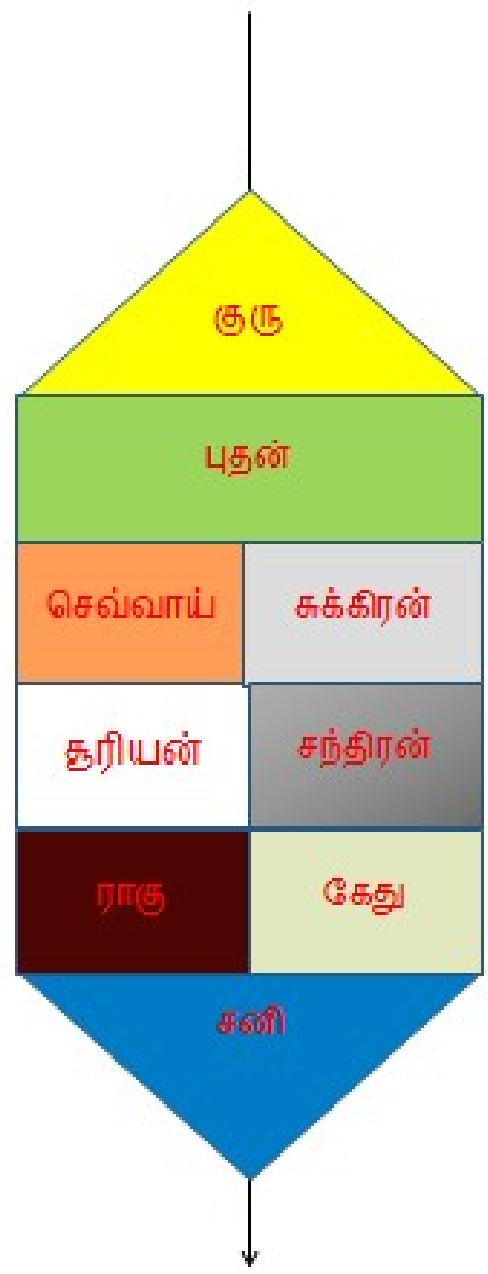

ஒரு உயிரை உருவாக்கும் தன்மையை கொண்டதால் குரு என்ற கோள் குழந்தை பருவத்தை குறிக்கிறது.

புதன் என்ற கோள், இளமை மற்றும் பள்ளி பருவத்தை குறிக்கிறது.

செவ்வாய் (அண் ), சுக்கிரன் (பெண்) பதின் மற்றும் திருமண பருவத்தை குறிக்கிறது.

சூரியனும் சந்திரனும், தந்தை மற்றும் தாயின் பருவத்தை குறிக்கிறது.

ராகுவும் கேதுவும், தாத்தா பாட்டி பருவத்தை குறிக்கிறது.

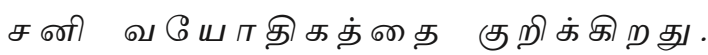
(Baskaran,2017,p.87) 
140 / தமிழ்ப் பேராய்வு ஆய்விதழ்

\section{தமிழ் சோதிடம் - ஒப்புமை}

சோதிடத்தின் அடிப்படை காலச்சக்கரம் / ராசிமானம். இந்த ராசிமானத்தின் அடிப்படை 12 ராசகள். அதுபோன்று தமிழின் அடிப்படை:

12 உயிர் எழுத்துக்கள்.

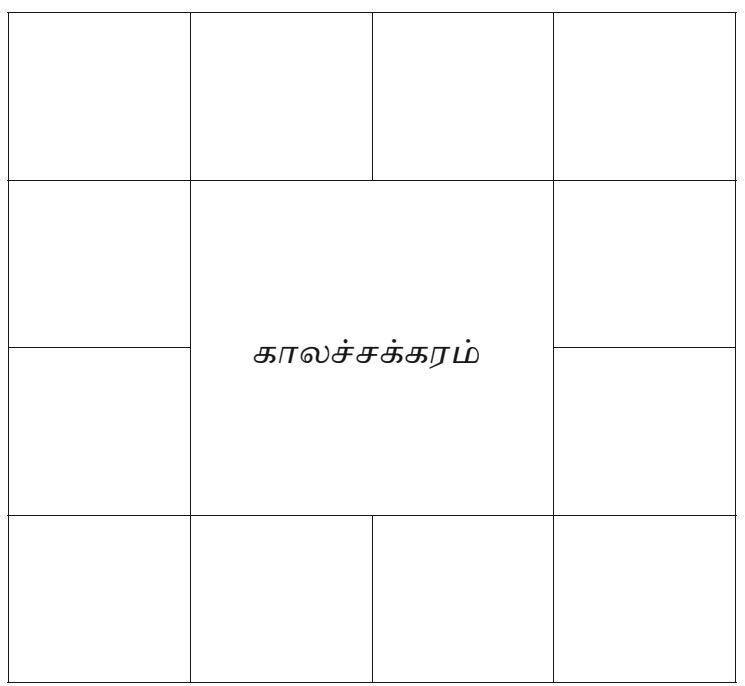

\begin{tabular}{|c|c|c|c|}
\hline ஒளா & அ & ஆ & இ \\
\hline ஓ & \multirow{2}{*}{\multicolumn{2}{|c|}{ காலச்சக்கரம் }} & ஈ \\
\hline ๑ & & & உ \\
\hline ஐ & ஏ & $\sigma$ & ஊ \\
\hline
\end{tabular}

மூலம்: https://en.wikipedia.org/wiki/Tamil grammar

\section{ஆண் பெண் ராசிகள் - குறில் நெடில்}

சே ா தி ட த் தி ல் ஆ ண் பெ ராசயைப்போல், தமிழ் மொழியில் குறில் நெடில் என்ற இந்த தன்மையே உயிர்
எழுத்துக்கள் உருவாக அடிப்படையாக உள்ளது.

\begin{tabular}{|c|c|c|c|}
\hline பெண் & ஆண் & பெண் & ஆண் \\
\hline ஆண் & \multirow{2}{*}{\multicolumn{2}{|c|}{ காலச்சக்கரம் }} & பெண் \\
\hline பெண் & & & ஆண் \\
\hline ஆண் & பெண் & ஆண் & பெண் \\
\hline
\end{tabular}

\begin{tabular}{|c|c|c|c|}
\hline நெடில் & குறில் & நெடில் & குறில் \\
\hline குறில் & \multirow{2}{*}{\multicolumn{2}{|c|}{ காலச்சக்கரம் }} & நெடில் \\
\hline நெடில் & & & ாறில் \\
\hline குறில் & நெடில் & குறில் & நெடில் \\
\hline
\end{tabular}

மூலம்: <https://en.wikipedia.org/wiki/Tamil_ grammar>

\section{தனி நிலை எழுத்து - ㅇ - வல்லினம் மெல்லினம் இடையினம்}

சோதிடத்தில் சரம், ஸ்திரம், உபயம் என்ற இயக்கத்தை போல், தமிழ் மொழியில் வல்லினம், மெல்லினம், இடையினம் என்ற இந்த தன்மையே மெய் எழுத்துக்களின் வகைகளைக் குறிக்கிறது.

\begin{tabular}{|c|c|c|c|}
\hline உபயம் & சரம் & ஸ்திரம் & உபயம் \\
\hline ஸ்திரம் & \multirow{2}{*}{\multicolumn{2}{|c|}{ காலச்சக்கரம் }} & சரம் \\
\hline சரம் & & & ஸ்திரம் \\
\hline உபயம் & ஸ்திரம் & சரம் & உபயம் \\
\hline
\end{tabular}




\section{தமிழ்ப் பேராய்வு ஆய்விதழ் / 141}

\begin{tabular}{|c|c|c|c|}
\hline $\begin{array}{l}\text { இடையினம் } \\
\text { உயிர்மெய் }\end{array}$ & $\begin{array}{c}\text { வல்லினம் } \\
\text { உயிர் }\end{array}$ & $\begin{array}{l}\text { மெல்லினம் } \\
\text { மெய் }\end{array}$ & $\begin{array}{l}\text { இடையினம் } \\
\text { உயிர்மெய் }\end{array}$ \\
\hline $\begin{array}{l}\text { மெல்லினம் } \\
\text { மெய் }\end{array}$ & \multirow{2}{*}{\multicolumn{2}{|c|}{ காலச்சக்கரம் }} & வல்லினம் உயிர் \\
\hline $\begin{array}{c}\text { வல்லினம் } \\
\text { உயிர் }\end{array}$ & & & $\begin{array}{l}\text { மெல்லினம் } \\
\text { மெய் }\end{array}$ \\
\hline $\begin{array}{l}\text { இடையினம் } \\
\text { உயிர்மெய் }\end{array}$ & $\begin{array}{l}\text { மெல்லினம் } \\
\text { மெய் }\end{array}$ & $\begin{array}{c}\text { வல்லினம் } \\
\text { உயிர் }\end{array}$ & $\begin{array}{l}\text { இடையினம் } \\
\text { உயிர்மெய் }\end{array}$ \\
\hline
\end{tabular}

மூலம்: https://en.wikipedia.org/wiki/Tamil_ grammar

\section{ஐந்திணை}

தமிழர்களின் ஐந்திணையே, சோதிடத்தின் பஞ்ச பூத தத்துவம். குறிஞ்ச முல்லை மருதம் நெய்தல் பாலை என்பதே நெருப்பு நிலம் காற்று நீர் ஆகாயம் (நட்சத்திரம்) என்ற தன்மையை உணர்த்துகிறது (Baskaran, 2017, p.60).

\begin{tabular}{|c|c|c|c|}
\hline நீர் & நெருப்பு & நிலம் & காற்று \\
\hline காற்று & \multirow{2}{*}{\multicolumn{2}{|c|}{ காலச்சக்கரம் }} & நீர் \\
\hline நிலம் & & & நெருப்பு \\
\hline நெருப்பு & நீர் & காற்று & நிலம் \\
\hline
\end{tabular}

எழுத்துக்களின் எண்ணிக்கை

12 ராசி என்ற நிலையிலிருந்து இன்று சோதிடம் 249 உப நட்சத்திரம் என்ற பரந்த நிலையை, விம்சோத்ரி திசாபுத்தி அடிப்படையில் அடைந்துள்ளது. அதாவது 27 நட்சத்திரத்தை மேலும் 9 பகுதியாக பிரிக்கும் போது அவை 243 உப நட்சத்திரமாக கிடைக்கும். இதில் இரண்டு உப நட்சத்திரம் 3 ராசிகளில் பகிர்ந்தளிக்கும் படி அமையும்.

அதேபோல், தமிழில் 12 உயிர் எழுத்துக்கள், 18 மெய் எழுத்துக்கள், 216 உயிர் மெய் எழுத்துக்கள் , 1 ஆய்த எழுத்து என்று 247
எழுத்துக்கள் உள்ளன. இவை வரி வடிவம் தாங்கிய எழுத்துக்கள். மேலும் ஒலி வடிவம் தாங்கி குற்றியலிகரம், குற்றியலுகரம் என்ற ஒலி அசைவுகளின் தன்மையோ (b) (உப நட்சத்திரத்தை 6 ராசிகளில் பகிர்ந்தளித்தது போல்) மொத்தம் 249 ஓலிகள் உள்ளன (Victor, 2012, p.107).

12 ராச என்ற தன்மையிலிருந்து 249 உப நட்சத்திரம் என்ற தன்மையைப்போல், 12 உயிர் எழுத்திலிருந்து 249 ஒலிகளின் தன்மை என்பது சோதிடம் தன்னிறைவை அடைந்ததே என்று கூறலாம் (Baskaran, 2017, p.17). இவ்வாறு எழுத்துக்களின் எண்ணிக்கை அதிகமாக உள்ளதால் தான் தமிழில் ஒரு வார்த்தையை அதே ஒலியோடு ஒலிக்க முடிகிறது உதாரணம். தமிழ் என்ற வார்த்தையே.

சோதிடத்தில் 249 உப நட்சத்திரம் இருந்தால் தான் துல்லியமான பலனை சொல்ல முடியும் என்பதை இயற்கை சுட்டிக்காட்டுகி $ற த ு$. தமிழும் சோதிடமும் இயற்கையை தழுவியது என்பதை இங்கு கவனிக்கவும். சோதிடத்தில்

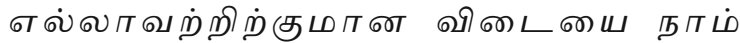
கா ல ச் ச க் கர த்தி லி ருந்து எ எு க் क றோ ம் என்பதை உணர்த்தும் வகையிலே “எல்லா சொற் களும் பொருள் குறித்தனவே " (தொல்காப்பியம், , சொல் 640) என்று தொல்காப்பியம் பறைச்சாற்றுகிறது (Victor, 2012, p.236).

\section{லக்னம்}

சோதிடம் என்பது வெளிச்சம் (சூரியன்) விழும் இடம். இதையே சோதிடத்தில் லக்னம் என்கிறோம். சோதிடத்தில் ஒருவரின் விதியை தீர்மானிப்பது லக்கினமே. அதேபோல் ஒரு எழுத்தாயினும், பல எழுத்துக்கள் சேர்ந்து ஒரு சொல்லாயினும், பல சொற்கள் சேர்ந்து பொருள் தரும் ஒரு சொற்றடர் ஆயினும், பல சொற்கள் சேர்ந்து ஒரு செய்யுள் ஆயினும், செய்யுளின் தொகுப்பாக அணி भயினும், இந்த அனைத்து நிலைகளில் தேர் ச்சி பெற்று புலமை என்ற ஒவ்வொரு நிலையில், சோதடடத்தில் ஒருவரின் தலை எழுத்தை லக்கினம் தர்மானிப்போதுபோல் தமிழில் விதிகளைத் தீர்மானிப்பது இலக்கணமே 
(Baskaran, 2002, p.42). இந்த லக்கினமும் இலக்கணமும், ஓன்று தான்.

சோதிடத்தில் பருவ காரகங்கள் மனிதனின் ஒவ்வொரு படிநிலையை கூறுகிறது. அதே போல் தமிழ் இலக்கணமும் இந்த படி நிலையை அப்படியே எடுத்துரைக்கிறது.

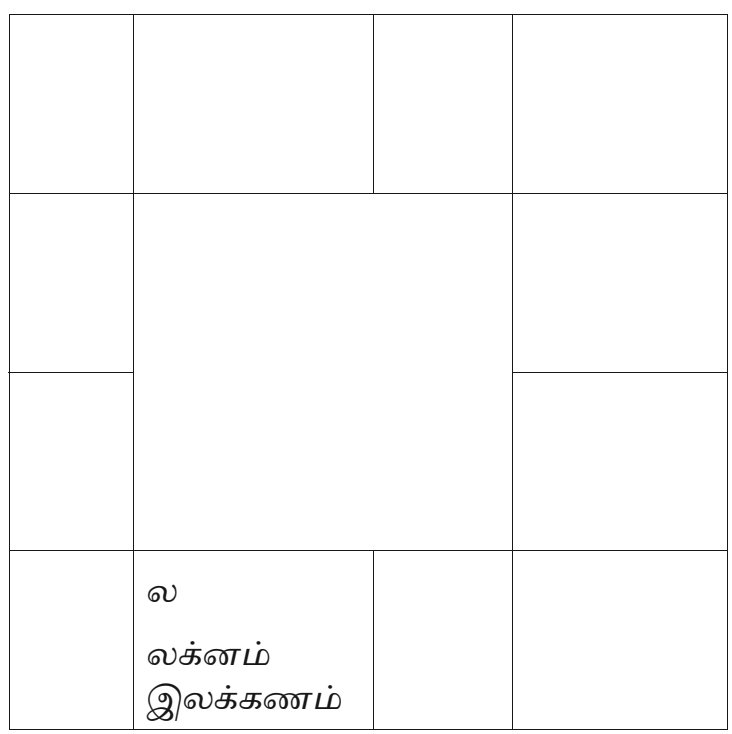

மூலம்: https://ta.wikipedia.org/wiki/தமிழ்_ இலக்கணம்

பருவ காரகங்கள்

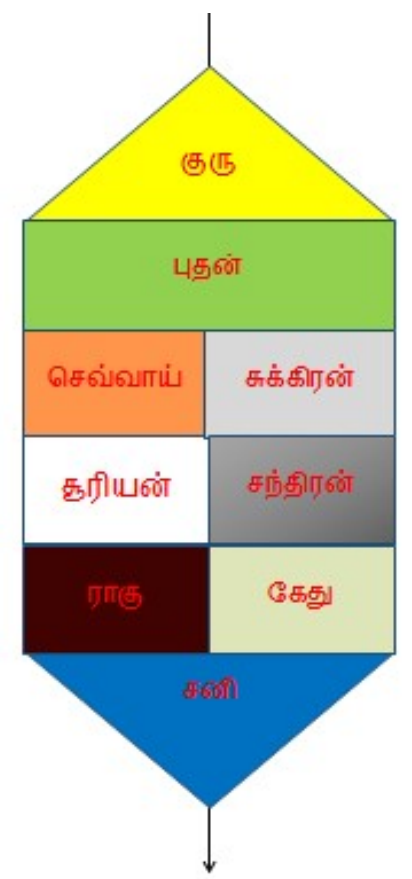

சோதிடத்தி ல் ஒவ் வொரு பருவ நிலையை அடைவதுபோல் எழுத்து என்ற அளவிலிருந்து, புலமை என்ற அளவிற்கு 6 படிநிலை இலக்கணத்தைக் கொண்டுள்ளது தமிழ் மொழி.

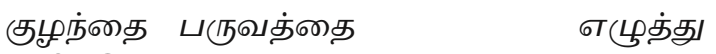
குறிக்கிறது.

இளமை மற்றும் பள்ளி பொருள் பருவத்தை குறிக்கிறது.

பதின் மற்றும் திருமண சொல் பருவத்தை குறிக்கிறது.

தந்தை மற்றும் தாயின் பருவத்தை குறிக்கிறது.

யாப்பு தாத்தா பாட்டி (செய்யுள்) பருவத்தை குறிக்கிறது. அணி வயோதிகத்தை குறிக்கிறது.

(Baskaran,2013,p.178)

மூலம்: https://ta.wikipedia.org/wiki/ அறுவகை_இலக்கணம்

\section{ஆண் பெண் ராசிகள் அகம்}

\section{(லக்னம்சார்ந்தது), புறம் (லக்னம்சாராதது)}

சசா தி ட த் தி ல் ஆ ண் பெ ராசயைப்போல், தமிழ் மொழியில் அகம் புறம் என்ற லக்னம் சார்ந்ததும், லக்னம் சாராததுமான இலக்கியங்களை (உதாரணம்: அகநானூறு புறநானூறு) கொண்டுள்ளது

\begin{tabular}{|l|l|l|l|}
\hline பெண் & \multirow{2}{*}{ भண் } & பெண் & भண் \\
\hline \multirow{2}{*}{ ஆண் } & \multicolumn{2}{|l|}{ कாலச்சக்கரம் } & பெண் \\
\cline { 1 - 3 } பெண் & \multicolumn{2}{|l|}{ ஆண் } \\
\hline \multirow{2}{*}{ ஆண் } & பெண் & ஆண் & பெண் \\
\hline
\end{tabular}




\begin{tabular}{|c|c|c|c|}
\hline புறம் & அகம் & புறம் & அகம் \\
\hline அகம் & \multirow{2}{*}{\multicolumn{2}{|c|}{ காலச்சக்கரம் }} & புறம் \\
\hline புறம் & & & அகம் \\
\hline அகம் & புறம் & அகம் & புறம் \\
\hline
\end{tabular}

மூ லம் : https://ta.wikipedia.org/wiki/ பொருள்_இலக்கணம்

\section{தமிழர்களின் வாழ்வியல்}

தமி ீர் கள் தங் கள து வ ா ம் வ இயற்கைய ஒட்டியே அமைத்து க் கொண்டனர். தமிழர் கள் முழு நிலவு நாளையே, மாதத்தின் முதல் நாளா க கொண்டிருந்தனர். அதனாலேயே சித்திரை நட்சத்திரத்தில் சந்திரன் வரும் முழு நிலவு (சூரியன் மேஷத்தில் இருக்கும் போது) எடுத்து கொண்டான். இவ்வாறாகவே தமிழ் மாதங்கள் உருவாகின. அதற்கு அவர்களின் சோதிட அறிவே காரணம்.

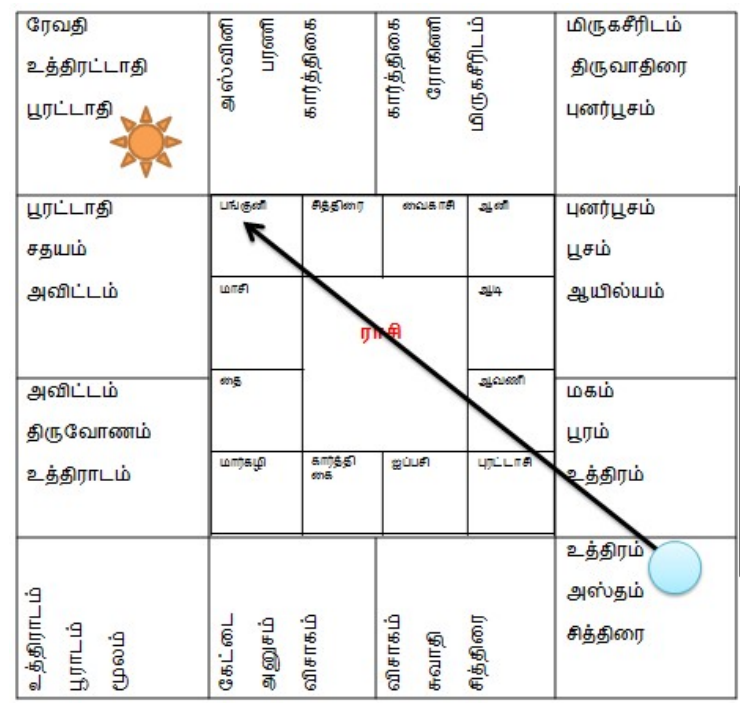

நாளை சித்திரை மாதம் என்று மேஷத்தில்

\begin{tabular}{|c|c|c|}
\hline மாதம் & தேதி & சறப்பு \\
\hline சத்திரை & 14 April - 13 May & $\begin{array}{l}\text { முழு நிலவு நாள்: சித்திரை. } \\
\text { சித்திரை திருவிழா, சித்ரா } \\
\text { பவுர்ணமி, வருட பிறப்பு }\end{array}$ \\
\hline வைகாச & 14 May - 14 June & $\begin{array}{c}\text { முழு நிலவு நாள் : விசாகம். } \\
\text { வைகாசி விசாகம் }\end{array}$ \\
\hline ஆனி & 15 June - 15 July & $\begin{array}{c}\text { முழூ நிலவு நாள் : அனுஷம். } \\
\text { ஆணி திருமஞ்சம் ஆணி } \\
\text { உத்திரம் }\end{array}$ \\
\hline ஆடி & 16 July - 16 August & $\begin{array}{c}\text { முழு நிலவு நாள் : } \\
\text { உத்திராடம். ஆடி வெள்ளி, } \\
\text { ஆடி அமாவாசை. அடி } \\
\text { பட்டம் தேடி விதை }\end{array}$ \\
\hline ஆவணி & 17 August - 16 September & $\begin{array}{l}\text { முழு. நிலவு நாள் : } \\
\text { அவிட்ம். அவணி } \\
\text { அவிட்டம் விநாயகர் } \\
\text { சதுர்த்தி }\end{array}$ \\
\hline புரட்டாசி & 17 September - 16 October & $\begin{array}{l}\text { முழு நிலவு நாள் : } \\
\text { உத்திரட்டாதி. அூயுத பூசை }\end{array}$ \\
\hline
\end{tabular}


144 / தமிழ்ப் பேராய்வு ஆய்விதழ்

\begin{tabular}{|c|c|c|}
\hline ஐப்பச & 17 October - 15 November & $\begin{array}{c}\text { முழு நிலவு நாள் : அஸ்வினி. } \\
\text { ஐப்பசி மழை ஐப்பசி } \\
\text { வெள்ளி த்பலளி தரருநாள் }\end{array}$ \\
\hline கார்த்திகை & 16 November - 15 December & $\begin{array}{l}\text { முழு நிலவு நாள் : } \\
\text { கார்த்திகை. திரு கார்த்திகை } \\
\text { கார்த்திகை பௌர்ணமி }\end{array}$ \\
\hline மார்கழி & 16 December - 13 January & $\begin{array}{c}\text { முழு நிலவு நாள் : } \\
\text { மிருகசாடம். அருட தரிசனம் }\end{array}$ \\
\hline தை & 14 January - 12 February & $\begin{array}{c}\text { முழு நிலவு நாள் : பூசம். தை } \\
\text { பூசம் }\end{array}$ \\
\hline மாச & 13 February - 13 March & $\begin{array}{c}\text { முழு நிலவு நாள் : மகம். } \\
\text { சவராத்திரி }\end{array}$ \\
\hline பங்குனி & 14 March - 13 April & $\begin{array}{l}\text { முழு நிலவு நாள் : உத்திரம். } \\
\text { பங்குனி உத்திரம் }\end{array}$ \\
\hline
\end{tabular}

மூலம்:https://en.wikipedia.org/wiki/Tamil_calendar/_Baskaran. 2017,p.46 / Victor, 2012,p.141

\section{முடிவுரை}

மேற்கூறிய கருத்துக்கள் மூலம், தமிழர்கள் ஒவ்வொரு மாதத்திலும், ஒரு வரலாற்று புதையலை வைத்துள் எ னர் என்பதை அறிய லா ம். இய இற்கயை தழுவிய தமிழர்களின் வாழ்வு, நம் தட்ப வெப்ப நிலை, வேளாண் பற்றிய அறிவு, அது சார்ந்த பண்டிகைகள் அனைத்தும் தமிழர்களே இந்த சோதிட கலையை உருவாக்கிர் என்பதற்கு சான்று. மேலும் இயற்கையை ஒட்டி அமையும் எந்த ஒரு கலையும் ஒத்த அமைப்பை கொண்டிருக்கும் என்றும், வெவ்வேறு காலகட்டத்தி ல் அவை பரிணமித்து காலக்கண்ணாடி போல் செயல்படும் என்பதும் திண்ணம். இதற்கு இயற்கையாய் உருவான தமிழ் மொழி எப்படி இயற்கையின் தன்மையை கூறும் சோதிடத்தோடு தொடர்பு கொண்டுள்ளது
என காண்க (Victor,2012,p.101). மேலும் தமிழ் என்ற மொழி எப்படி சூசகமாக, உப நட்சத்திரம் கொண்டே நாம் சோதிடத்தில் நிறைவு நிலை அடைய முடியும் என்று கூறுவதையும் காண்க.

இயற்கை=தமிழ்=சோதிடம். தமிழ் என்ற சொல் அமிழ்து என்ற சொல்லிலிருந்து உருவானது (Victor, 2012, p.93). அமிழ்து உண் ட வ னு க் மு மர ம் இ ல் ல என்பதால். (புவி உள்ள அளவும் தமிழ் இருக்கும் என்பதைவிட) தமிழ் உள் ள அளவும் இந்த புவி இருக்கும் என நம்மை பெருமிதம் கொள்ள செய்வது இந்த “தமிழும் சோதிடமும்”. தமிழிற்கும், இயற்கைக்கும், பாஸ்கரா சோதிடத்திற்கும் உள்ள மேலும் பல வியக்கத்தக்க தொடர்புகளோடு மீண்டும் இன்னொரு கட்டுரையில் சந்திப்போம்.

\section{References}

Aruvagai Ilakkanam. (2011). In Wikipedia. Retrieved from https://ta.wikipedia.org/wiki/ அறுவக__இலக்கணம்

Baskaran, K. (2013a). The Secrets of Baskara Astrology Part 1. Madurai: Thilak Astro Books.

Baskaran, K. (2013b). The Secrets of Baskara Astrology Part 2. Madurai: Thilak Astro Books. 
Baskaran, K. (2017a). Baskara Jodhida Varudamalar. Madurai: Thilak Astro Books.

Baskaran, K. (2017b). Baskara Naveena Natchathira Jodhidam. Madurai: Thilak Astro Books.

Baskaran, K. (2017c). Panchangam Baskara Jodhida Varuda malar. Madurai: Thilak Astro Books.

Baskaran,K. (2005). Astrologics of Kaalchakra. Madurai: Mother Meera Publication.

Baskaran,K. (2010). Baskara Jodhida Ragasiyam. Madurai: Thilak Astro Books.

Porul Ilakkanam. (2019). In Wikipedia. Retrieved from https://ta.wikipedia.org/wiki/ பாெரள்டஇலக்கணம்

Tamil Grammar. (2019) In Wikipedia. Retrieved from https://en.wikipedia.org/wiki/Tamil_ grammar

Victor, Ma. So. (2012). Kumarikkandam. Ariyalur: Yaathisai Pathipagam.

Victor, Ma. So. (2012). Seyyan. Ariyalur: Yaathisai Pathipagam.

Victor, Ma. So. (2018). Ulagin Muthal Semmoli, Tamilil. Ariyalur: Yaathisai Pathipagam. 